\title{
HCG Trigger After Failed GnRH Agonist Trigger Resulted in Two Consecutive Live Births: A Case Report
}

\begin{abstract}
Sara Liest ${ }^{1 *}$, Iben Riishede Christiansen ${ }^{2,3}$, Lisbeth Prætorius ${ }^{4}$, Jeanette Bogstad ${ }^{5}$, Nina la Cour Freiesleben ${ }^{3,4}$, Anja Pinborg ${ }^{3,5}$ and Kristine Løss/ ${ }^{5 *}$

${ }^{1}$ Department of Obstetrics and Gynecology, North Zealand Hospital, Hillerød, Denmark, ${ }^{2}$ Center of Fetal Medicine and Pregnancy, Department of Obstetrics, Copenhagen University Hospital Rigshospitalet, Copenhagen, Denmark, ${ }^{3}$ Institute of Clinical Medicine, Faculty of Health and Medical Sciences, University of Copenhagen, Copenhagen, Denmark, ${ }^{4}$ The Fertility Clinic, Department of Obstetrics and Gynecology, Copenhagen University Hospital Hvidovre, Hvidovre, Denmark, ${ }^{5}$ The Fertility Clinic, Copenhagen University Hospital Rigshospitalet, Copenhagen, Denmark
\end{abstract}

OPEN ACCESS

Edited by:

Jan Tesarik

MARGen Clinic, Spain

Reviewed by:

Ali Abbara,

Imperial College London,

United Kingdom

Talya Shaulov,

University of Montreal Hospital Centre

(CRCHUM), Canada

${ }^{*}$ Correspondence:

Sara Liest

sara.liest@hotmail.com

Kristine Løss

kristine.loess/@regionh.dk

Specialty section

This article was submitted to

Assisted Reproduction,

a section of the journal

Frontiers in Reproductive Health

Received: 25 August 2021 Accepted: 27 September 2021

Published: 27 October 2021

Citation

Liest S, Riishede Christiansen I, Prætorius L, Bogstad J, Freiesleben NIC, Pinborg A and Løss/ K (2021) HCG Trigger After Failed GnRH Agonist Trigger Resulted in Two Consecutive Live Births.

A Case Report.

Front. Reprod. Health 3:764299.

doi: 10.3389/frph.2021.764299
Background: Failed gonadotropin-releasing hormone $(\mathrm{GnRH})$ agonist trigger with no oocyte retrieved during aspiration of several follicles is a rare but recurrent situation that can be rescued by the termination of the aspiration procedure, retriggering by human chorion gonadotropin (hCG), and repeated oocyte pickup $36 \mathrm{~h}$ later. Failed $\mathrm{GnRH}$ agonist trigger is frustrating and unsatisfactory, and fertility doctors must be aware of possible hCG retriggering and retained opportunity for successful cycle outcome.

Objective: In this case report, we present a woman who experienced failed $\mathrm{GnRH}$ agonist trigger and rescue hCG retrigger followed by two consecutive live births after frozen-thawed single blastocyst transfers.

Methods: A case report.

Results: Two healthy children were born in 2018 and 2020, respectively as a result of controlled ovarian stimulation for IVF, failed GnRH agonist trigger followed by hCG re-trigger, and successful retrieval of 25 oocytes.

Conclusion: Retriggering with hCG after failed $\mathrm{GnRH}$ agonist trigger can result in consecutive live births, and such knowledge can prevent cycle cancellation and patient discouragement. Knowledge on retriggering with hCG and consecutive live births after failed $\mathrm{GnRH}$ agonist trigger can prevent cycle cancellation and patient discouragement.

Keywords: empty follicle syndrome (EFS), rescue hCG, failed agonist trigger, re-trigger with hCG, consecutive live births after empty follicle syndrome

\section{INTRODUCTION}

Ovarian hyperstimulation syndrome (OHSS) is a serious complication to in vitro fertilization (IVF), which is potentially life-threatening. Moderate to severe OHSS has a prevalence of 3-8\% $(1,2)$ being highest for women with polycystic ovarian syndrome (PCOS). The risk of OHSS increases with an increasing number of growing follicles and also high oestradiol levels on the day of ovulation trigger, and it increases with an increased number of retrieved oocytes $(3,4)$. More than 18 growing 
follicles $\geq 11 \mathrm{~mm}$ on the day of ovulation trigger is a good predictor of severe OHSS $(5,6)$. The live birth rate increases with number of oocytes retrieved up to 15 in fresh IVF cycles and then plateaued (4), whereas the cumulative live birth rate steadily increases with number of oocytes reaching $70 \%$ when 25 oocytes were retrieved (7).

In the GnRH antagonist protocol, the risk of OHSS is significantly reduced when final oocyte maturation is triggered by a $\mathrm{GnRH}$-agonist ( $\mathrm{GnRHa}$ ), which stimulates the endogenous pituitary secretion of luteinizing hormone (LH), instead of the traditionally used human chorionic gonadotropin (hCG) trigger (8). hCG provides LH activity with a much longer half-life than endogenous $\mathrm{LH}$ and promotes the production of vascular endothelial growth factor (VEGF) by endothelial cells (9), which is thought to be the principal vasoactive mediator causing the increased vascular permeability seen in OHSS.

The GnRHa trigger has been introduced as the trigger of choice in women at risk of OHSS, which is easily judged based on the number of growing follicles on trigger day during the standard transvaginal ultrasound examination that is always performed during IVF. The use of GnRHa trigger does not hamper the yield of mature oocytes (10). Another strategy used to reduce the risk of OHSS is to freeze all developed embryos to avoid pregnancy-related endogenous hCG production. Postponed embryo transfer to a subsequent natural or artificial frozen-thawed embryo transfer does not decrease the chance of ongoing pregnancy (11-13).

The administration of a GnRHa initiates the endogenous surge of LH that is mandatory for the final oocyte maturation proceeding ovulation. However, a suboptimal LH response can reduce or inhibit oocyte yield during oocyte retrieval (14). Failed GnRHa trigger, also called empty follicle syndrome (EFS), is defined as the failure to retrieve oocytes from preovulatory follicles under oocyte pickup despite flushing. EFS has been reported with an incidence ranging from $1.0 \%$ to $3.5 \%$ after the use of GnRHa trigger (15-17). The use of hCG as a rescue trigger administered $36 \mathrm{~h}$ before a second oocyte retrieval has been described in other cases $(18,19)$, but might not be common knowledge among all the doctors involved in fertility treatment.

The present case is important to provide knowledge about the possibility of consecutive live births even in cycles with failed GnRHa trigger, if handled properly. Correct counseling is very helpful in clinical situations where this unexpected situation appears. To the best of our knowledge, two consecutive live births as a result of hCG retrigger have not previously been reported.

Informed written consent has been obtained from the woman for publication of the case report.

\section{CASE DESCRIPTION}

In 2016, a 29-year old, nulligravida, healthy woman and her partner were referred to a tertiary clinic because of combined infertility and unsuccessful intrauterine inseminations. The duration of infertility was 1 year. The female diagnosis was PCOS based on secondary amenorrhea and an antral follicle count of 80 corresponding to an antimüllarian hormone (AMH) level of
$96 \mathrm{pmol} / \mathrm{L}$. Androgen status, prolactin, and thyroid stimulating hormone levels were normal. Follicle stimulating hormone (FSH) and $\mathrm{LH}$ levels were $5.8 \mathrm{IU} / \mathrm{L}$ and $\mathrm{LH} 6.7 \mathrm{IU} / \mathrm{L}$, respectively. BMI was $21 \mathrm{~kg} / \mathrm{m}^{2}$. The sperm volume and count were just within the normal range, but the motility was affected with a lower than a normal number of progressively motile sperm cells according to the recommendation of ICSI.

The woman underwent a total of three controlled ovarian stimulation cycles that are summarized in Table 1. All three were in a flexible GnRH antagonist protocol. In the first two stimulations, final oocyte maturation was triggered with hCG as the risk of OHSS was not pending based on the number of follicles $>11 \mathrm{~mm}$. After the first ovarian stimulation 11 oocytes were retrieved, but none of them developed into blastocysts. In the second stimulation, 12 oocytes were retrieved, one cleavage stage embryo was transferred, and the last embryos did not develop into blastocysts. None of the first two simulations resulted in pregnancy.

In the third ovarian stimulation, a daily dose of human menopausal gonadotropin (Menopur; Ferring Pharmaceuticals) 112-131 IU was administered from cycle day 2 (CD2), and $0.25 \mathrm{mg}$ of $\mathrm{GnRH}$ antagonist (Cetrotide; Merck) was added daily when the leading follicles reached 13-14 mm (stimulation day 12). On stimulation day 16 , when $\sim 15$ follicles reached $17 \mathrm{~mm}$, and a total of 23 follicles were $\geq 11 \mathrm{~mm}$, the final oocyte maturation was triggered with $0.50 \mathrm{mg}$ of $\mathrm{GnRH}$ agonist Suprefact (Buserelin; Orifarm). Elective freeze-all was planned to reduce the risk of OHSS. Oocyte retrieval was performed $36 \mathrm{~h}$ after trigger using ultrasound-guided transvaginal needle aspiration after administration of local anesthetic and low-dose intravenous opioids. Approximately 12 follicles from the right ovary were emptied without retrieval of any oocytes, even though flushing was used in several follicles. The procedure was stopped, and five follicles in the right ovary and all the follicles in the left ovary were left untouched. No GnRH antagonist was administered after the oocyte retrieval. Rescue hCG retrigger was performed at $10 \mathrm{pm}$ the same day with chorion gonadotropin-alfa (Ovitrelle; Merck) $250 \mu \mathrm{g}$, and $36 \mathrm{~h}$ later a second oocyte retrieval was performed. Here 25 oocytes, of which 20 were mature, were retrieved from 30 follicles, 13 were fertilized and five-six days after the second oocyte retrieval, eight good quality blastocysts were vitrified. There were no symptoms of OHSS after rescue hCG retrigger.

The patient used contraceptive pills for 1 month and consecutive frozen-thawed embryo transfer (FET) was planned in artificial hormone replacement cycles (AC). In the fourth AC FET the patient conceived and delivered a healthy girl at gestational age $40+0$ weeks, 4 days after initiation of partus provocatus medicamentalis by oral misoprostol. The weight of the girl was $2,155 \mathrm{~g}(-34 \%)$. In the AC FET, the patient took $4 \mathrm{mg}$ oestradiol twice daily for 17 days before the addition of $90 \mathrm{mg}$ of vaginal progesterone gel (Crinone; Orifarm) daily. Single blastocyst warming and transfer were performed on the 6th day of progesterone.

After 18 months, the couple returned with the wish for a second child. There were still four vitrified blastocysts left. In the third and last AC FET cycle (one blastocyst did not survive 
TABLE 1 | Overview of the three controlled ovarian stimulation cycles and associated outcome.

\begin{tabular}{|c|c|c|c|}
\hline Treatment number (date of oocyte retrievals) & 1. $(16 / 112016)$ & 2. $(15 / 2$ 2017) & 3. $(1 / 4+/ 3 / 42017)$ \\
\hline Days of stimulation & 21 & 31 & 15 \\
\hline Gonadotropin starting dose & Menopur $75 \mathrm{IU}$ & Puregon $91 \mathrm{IU}$ & Menopur $112 \mathrm{IU}$ \\
\hline Gonadotropin total dose & $1,872 \mathrm{IU}$ & 3,295 IU & $2,051 \mathrm{IU}$ \\
\hline Fertilization method & ICSI & IVF/ICSI 6/6 oocytes & ICSI \\
\hline Number of oocytes & 11 & 12 & $(0) / 25^{\star}$ \\
\hline Number of mature oocytes & 5 & NA/3 & 20 \\
\hline Number of cleavage stage embryos & 4 & $0 / 2$ & 13 \\
\hline Number of blastocysts ${ }^{\star \star}$ & 0 & 0 & 8 \\
\hline Embryos transferred in the stimulated cycle & 0 & 1 & 0 \\
\hline Number of FET cycles & 0 & 0 & $7^{\star \star \star}$ \\
\hline Pregnancies & 0 & 0 & 2 \\
\hline Live births & 0 & 0 & $2(2018$ + 2020) \\
\hline
\end{tabular}

`Zero oocytes were retrieved after GnRHa trigger, after rescue hCG re-trigger 25 oocytes were retrieved.

${ }^{* *}$ Gardner score (on Day 5 or 6) $\geq 3 B B$.

${ }^{* \star \star}$ One blastocyst underwent atresia after warming.

warming), the patient conceived again and delivered a healthy boy at gestational age $40+0$ weeks, weight $2,900 \mathrm{~g}$ and length $52 \mathrm{~cm}$. In the second pregnancy, the patient was treated with acetylsalicylic acid $150 \mathrm{mg}$ daily from gestational age 12 weeks because of IUGR in first pregnancy.

In our case, rescue hCG retrigger, given after a failed GnRHa trigger and EFS, and a second oocyte retrieval turned out to be a successful treatment with 25 oocytes retrieved, eight blastocysts suitable for vitrification, and finally two pregnancies and live births.

\section{DISCUSSION}

To our knowledge, we report for the first time a case of failed GnRHa trigger that ended up as a successful ICSI treatment with two consecutive live births as a result of rescue hCG re trigger. This was possible due to awareness of the phenomenon and interruption of the oocyte retrieval procedure in due time to perform hCG retrigger, followed by a second oocyte retrieval. The case is important in order to provide knowledge about consecutive live births even in cycles with failed GnRHa trigger if handled properly. Correct counseling from clinicians to patients is needed in clinical situations where this unexpected situation appears.

Several risk factors of failed agonist trigger have been identified: low BMI, low baseline LH, high total dose of administered gonadotropins, hypogonadotropic hypogonadism, or iatrogenic-induced pituitary downregulation in the long GnRHa protocol or after prolonged use of oral contraceptives (20). Patients with BMI $<22 \mathrm{~kg} / \mathrm{m}^{2}$ have a twice as high failure rate as patients with higher BMI $(P=0.039)$, baseline $\mathrm{LH}<2$ IU/L has a statistically significant higher failure rate compared to patients with a baseline $\mathrm{LH} \geq 2 \mathrm{IU} / \mathrm{L}$ (5.6 vs. $1.8 \%, P=0.048$ ), and patients who received $>3,800$ IU gonadotropins during stimulation had a 3.5 times higher failure rate compared with patients receiving $<3,800 \mathrm{IU}(P<0.0001)$ (19).
In the current case, there were no issues regarding patient compliance, and most likely the reason for failed GnRHa trigger was a suboptimal endogenous LH surge. Even though gonadotropin levels were measured and found within the midnormal range as a part of the standard fertility work-up of this anovulatory women (LH $6.7 \mathrm{IU} / \mathrm{L})$, no $\mathrm{LH}$ measurement was performed at the initiation (baseline) of this third ICSI treatment, which is a limitation to causal interpretation. However, the patient did use oral contraceptive pills (OCP) to initiate her bleeding, and the baseline LH level may have been suppressed.

As the incidence of EFS is generally low (15-17), and the sensitivity of baseline LH levels to predict EFS is low (15), we would suggest measurement of baseline LH level "on indication" rather than on all patients. Such indications could be the use of OCP or other drugs that induce pituitary downregulation even after a wash-out period and/or anovulation, especially if the anovulation is not a part of a PCOS diagnosis where LH levels are usually normal-high. If baseline LH is low after OCP, postponement of stimulation start could be applied if only small antral follicles (proceeded by another LH measurement), or a cautious gonadotropin dose for ovarian stimulation could be chosen aiming for hCG trigger with a minimal risk of OHSS.

In the present case, supplementary GnRH antagonist was not used after the first failed oocyte retrieval. We would not expect premature ovulation to be an issue in cases of genuine EFS. The initial GnRHa trigger is expected to empty the pituitary LH, and if this endogenous LH should reach a circulatory "threshold level" of concern, we would expect a suboptimal response with at least a few oocytes retrieved at the first retrieval rather than zero oocytes (genuine EFS).

Another question is whether to measure the LH level in blood or urine in the morning after the GnRHa trigger. Interestingly, it has recently been demonstrated that a standard urinary LH test on the morning after GnRHa trigger can be used by patients at home as an easy and convenient way to document a sufficient $\mathrm{LH}$ release in response to GnRHa trigger (21). Only three out 
of $359(0.8 \%)$ urine $\mathrm{LH}$ tests were negative; however, in one out of the three cases, an LH measurement in blood showed $\mathrm{LH}$ rise consistent with optimal response to the GnRHa trigger (21). Thus, the predictive value of a negative urine LH test does not seem reliable and could have resulted in ovulation if hCG retriggering and rescheduling of the oocyte retrieval had been based on the urine LH testing alone.

In our opinion, insufficient $\mathrm{LH}$ rise after $\mathrm{GnRHa}$ trigger is too uncommon to justify posttrigger $\mathrm{LH}$ measurements. Instead, if no oocytes are retrieved after proper emptying of $\sim 7$ preovulatory follicles, we advocate hCG retrigger and a second oocyte retrieval, as presented in this case report.

The diagnosis EFS could be given when no oocytes are retrieved from $\sim 7$ preovulatory follicles despite proper emptying and flushing. The risk of OHSS has been suggested to be reduced after failed GnRHa and hCG retrigger due to reduction of the oestradiol level after the first attempt to retrieve oocytes (18) as well as by an elective freeze-all regimen. Moderate to severe OHSS after rescue hCG retrigger has nevertheless been described, and an individual estimation of OHSS risk before hCGretrigger is important in each patient $(19,22)$. In this case report, we lack data on posttrigger serum LH, progesterone, and oestradiol.

The experience from the presented case report is that rescue hCG retrigger after failed GnRHa trigger can result in a complete family formation. The case is important as knowledge on retriggering with hCG and consecutive live

\section{REFERENCES}

1. Mourad, S, Brown J, Farquhar C. Interventions for the prevention of OHSS in ART cycles: an overview of cochrane reviews. Cochrane Database Systemat Rev. (2017) 1:CD012103. doi: 10.1002/14651858.CD012103.pub2

2. Toftager M, Bogstad J, Bryndorf T, Løssl K, Roskær J, Holland T, et al. Risk of severe ovarian hyperstimulation syndrome in $\mathrm{GnRH}$ antagonist versus $\mathrm{GnRH}$ agonist protocol: RCT including 1050 first IVF/ICSI cycles. Hum Reprod. (2016) 31:1253-64. doi: 10.1093/humrep/dew051

3. Tarlatzis BC, Griesinger G, Leader A, Rombauts L, Ijzerman-Boon PC, Mannaerts BM. Comparative incidence of ovarian hyperstimulation syndrome following ovarian stimulation with corifollitropin alfa or recombinant FSH. Reprod Biomed Online. (2012) 24:410-9. doi: 10.1016/j.rbmo.2012.01.005

4. Steward RG, Lan L, Shah AA, Yeh JS, Price TM, Goldfarb JM, et al. Oocyte number as a predictor for ovarian hyperstimulation syndrome and live birth: an analysis of 256,381 in vitro fertilization cycles. Fertil Steril. (2014) 101:96773. doi: $10.1016 /$ j.fertnstert.2013.12.026

5. Papanikolaou EG, Pozzobon C, Kolibianakis EM, Camus M, Tournaye H, Fatemi HM, et al. Incidence and prediction of ovarian hyperstimulation syndrome in women undergoing gonadotropin-releasing hormone antagonist in vitro fertilization cycles. Fertil Steril. (2006) 85:112-20. doi: 10.1016/j.fertnstert.2005.07.1292

6. Griesinger G, Verweij PJ, Gates D, Devroey P, Gordon K, Stegmann BJ, et al. Prediction of ovarian hyperstimulation syndrome in patients treated with Corifollitropin alfa or rFSH in a GnRH antagonist protocol. PLoS ONE. (2016) 11:e0149615. doi: 10.1371/journal.pone.0149615

7. Polyzos NP, Drakopoulos P, Parra J, Pellicer A, Santos-Ribeiro S, Tournaye $\mathrm{H}$, et al. Cumulative live birth rates according to the number of oocytes retrieved after the first ovarian stimulation for in vitro fertilization/intracytoplasmic sperm injection: a multicenter multinational analysis including $\sim 15,000$ women. Fertil Steril. (2018) 110:661-70.e1. doi: 10.1016/j.fertnstert.2018.04.039 births after failed GnRHa can prevent cycle cancellation and patient discouragement. Correct counseling from clinicians to patients is needed in clinical situations where this unexpected situation appears.

Perspective of the patient: Sharing the knowledge that hCG retrigger after failed GnRHa trigger is possible can prevent cycle cancellation and patient discouragement and result in consecutive live births.

\section{DATA AVAILABILITY STATEMENT}

The original contributions presented in the study are included in the article/supplementary material, further inquiries can be directed to the corresponding author/s.

\section{ETHICS STATEMENT}

Informed written consent has been obtained from the woman for publication of the case report.

\section{AUTHOR CONTRIBUTIONS}

IR, LP, JB, NF, AP, and KL helping with litterature, references, and reading correture on article. All authors contributed to the article and approved the submitted version.
8. Davenport M, Beverley V, Talmor AJ. Gonadotropin-releasing hormoneagonist triggering and a freeze-all approach: the final step in eliminating ovarian hyperstimulation syndrome? Obstet Gynecol Surv. (2017) 72:296-308. doi: 10.1097/OGX.0000000000000432

9. Albert C, Garrido N, Mercader A, Rao CV, Remohí J, Simón C, et al. The role of endothelial cells in the pathogenesis of ovarian hyperstimulation syndrome. Mol Hum Reprod. (2002) 8:409-18. doi: 10.1093/molehr/8.5.409

10. Engmann L, Benadiva C, Humaidan P. GnRH agonist trigger for the induction of oocyte maturation in GnRH antagonist IVF cycles: a SWOT analysis. Reprod Biomed Online. (2016) 32:274-85. doi: 10.1016/j.rbmo.2015. 12.007

11. Stormlund S, Sopa N, Zedeler A, Bogstad J, Prætorius L, Nielsen HS, et al. Freeze-all versus fresh blastocyst transfer strategy during in vitro fertilisation in women with regular menstrual cycles: multicentre randomised controlled trial. BMJ. (2020) 370:m2519. doi: 10.1136/bmj.m2519

12. Roque M, Haahr T, Geber S, Esteves SC, Humaidan P. Fresh versus elective frozen embryo transfer in IVF/ICSI cycles: a systematic review and metaanalysis of reproductive outcomes. Hum Reprod Update. (2019) 25:2-14. doi: 10.1093/humupd/dmy033

13. Ovarian Stimulation TEGGO, Bosch E, Broer S, Griesinger G, Grynberg M, Humaidan P, et al. ESHRE guideline: ovarian stimulation for IVF/ICSI. Hum Reprod Open. (2020) 2020:hoaa009. doi: 10.1093/hropen/hoaa009

14. Chen SL, Ye DS, Chen X, Yang XH, Zheng HY, Tang Y, et al. Circulating luteinizing hormone level after triggering oocyte maturation with $\mathrm{GnRH}$ agonist may predict oocyte yield in flexible GnRH antagonist protocol. Hum Reprod. (2012) 27:1351-6. doi: 10.1093/humrep/des049

15. Popovic-Todorovic B, Santos-Ribeiro S, Drakopoulos P, De Vos M, Racca A, Mackens S, et al. Predicting suboptimal oocyte yield following GnRH agonist trigger by measuring serum $\mathrm{LH}$ at the start of ovarian stimulation. Hum Reprod. (2019) 34:2027-35. doi: 10.1093/humrep/dez132

16. Christopoulos G, Vlismas A, Barsoum-Derias E, El-Shawarby S, Trew G, Lavery S. Rescue hCG to treat empty follicle syndrome after the use of a GnRH agonist as oocyte maturation trigger: first report on fresh 
embryo transfer and clinical pregnancy. Hum Fertil. (2015) 18:248-52. doi: 10.3109/14647273.2015.1071500

17. Castillo JC, Garcia-Velasco J, Humaidan P. Empty follicle syndrome after GnRHa triggering versus hCG triggering in COS. J Assist Reprod Genet. (2012) 29:249-53. doi: 10.1007/s10815-011-9704-8

18. Asada Y, Itoi F, Honnma H, Takiguchi S, Fukunaga N, Hashiba Y, et al. Failure of GnRH agonist-triggered oocyte maturation: its cause and management. $J$ Assist Reprod Genet. (2013) 30:581-5. doi: 10.1007/s10815-013-9966-4

19. Chang FE, Beall SA, Cox JM, Richter KS, DeCherney AH, Levy MJ. Assessing the adequacy of gonadotropin-releasing hormone agonist leuprolide to trigger oocyte maturation and management of inadequate response. Fertil Steril. (2016) 106:1093-100.e3. doi: 10.1016/j.fertnstert.2016.06.013

20. Humaidan P, Kol S. Suboptimal response to GnRH agonist trigger: causes and practical management. Curr Opin Obstet Gynecol. (2021) 33:213-7. doi: $10.1097 /$ GCO.0000000000000701

21. Cozzolino M, Matey S, Alvarez A, Toribio M, López V, Perona M, et al. Self-detection of the LH surge in urine after GnRH agonist trigger in IVFhow to minimize failure to retrieve oocytes. Front Endocrinol. (2020) 11:221. doi: $10.3389 /$ fendo.2020.00221

22. Lu X, Hong Q, Sun L, Chen Q, Fu Y, Ai A, et al. Dual trigger for final oocyte maturation improves the oocyte retrieval rate of suboptimal responders to gonadotropin-releasing hormone agonist. Fertil Steril. (2016) 106:1356-62. doi: $10.1016 /$ j.fertnstert.2016.07.1068

Conflict of Interest: The authors declare that the research was conducted in the absence of any commercial or financial relationships that could be construed as a potential conflict of interest.

Publisher's Note: All claims expressed in this article are solely those of the authors and do not necessarily represent those of their affiliated organizations, or those of the publisher, the editors and the reviewers. Any product that may be evaluated in this article, or claim that may be made by its manufacturer, is not guaranteed or endorsed by the publisher.

Copyright () 2021 Liest, Riishede Christiansen, Protorius, Bogstad, Freiesleben, Pinborg and Løssl. This is an open-access article distributed under the terms of the Creative Commons Attribution License (CC BY). The use, distribution or reproduction in other forums is permitted, provided the original author(s) and the copyright owner(s) are credited and that the original publication in this journal is cited, in accordance with accepted academic practice. No use, distribution or reproduction is permitted which does not comply with these terms. 